Research Paper

\title{
Reactive Oxygen Species Are Involved in Regulating Hypocontractility of Mesenteric Artery to Norepinephrine in Cirrhotic Rats with Portal Hypertension
}

\author{
Wei Chen*, De-Jun Liu*, Yan-Miao Huo, Zhi-Yong Wu, Yong-Wei Sun ${ }^{凶}$ \\ Department of Surgery, Ren Ji Hospital, School of Medicine, Shanghai Jiao Tong University, Shanghai 200127, China. \\ ${ }^{*}$ Wei Chen and De-Jun Liu are the co-first authors. \\ $\triangle$ Corresponding author: Yong-Wei Sun, Department of Surgery, Ren Ji Hospital, School of Medicine, Shanghai Jiao Tong University, \\ Shanghai 200127, China. E-mail: syw0616@126.com Tel: +86-021-68383713. \\ (c) Ivyspring International Publisher. This is an open-access article distributed under the terms of the Creative Commons License (http://creativecommons.org/ \\ licenses/by-nc-nd/3.0/). Reproduction is permitted for personal, noncommercial use, provided that the article is in whole, unmodified, and properly cited.
}

Received: 2013.1I.07; Accepted: 2014.02.18; Published: 2014.03.15

\begin{abstract}
Background: Oxidative stress is involved in the hypocontractility of visceral artery to vasoconstrictors and formation of hyperdynamic circulation in cirrhosis with portal hypertension. In the present study, we investigated the effect of reactive oxygen species (ROS) on the mesenteric artery contractility in $\mathrm{CCl}_{4}$-induced cirrhotic rats, and the roles of $\mathrm{G}$ protein-coupled receptors (GPCRs) desensitization and RhoA /Rho associated coiled-coil forming protein kinase (ROCK) pathways.

Methods: The mesenteric artery contraction to norepinephrine (NE) was determined by vessel perfusion system following treatments with apocynin, tempol or PEG-catalase. The protein expression of $\alpha$ I adrenergic receptor, $\beta$-arrestin-2, ROCK-I, moesin and p-moesin was measured by western blot. The interaction between $\alpha$ l adrenergic receptor and $\beta$-arrestin- 2 was assessed by co-immunoprecipitation.

Results: Pretreatment with apocynin or PEG-catalase in cirrhotic rats, the hydrogen peroxide level in the mesenteric arteriole was significantly decreased, and the dose-response curve of mesenteric arteriole to NE moved to the left with $\mathrm{EC}_{50}$ decreased. There was no significant change for the expression of $\alpha \mathrm{l}$ adrenergic receptor. However, the protein expression of $\beta$-arrestin- 2 and its affinity with $\alpha \mathrm{I}$ adrenergic receptor were significantly decreased. The ROCK-I activity and anti- Y-27632 inhibition in cirrhotic rats increased significantly with the protein expression unchanged. Such effects were not observed in tempol-treated group.

Conclusion: The $\mathrm{H}_{2} \mathrm{O}_{2}$ decrease in mesenteric artery from rats with cirrhosis resulted in down regulation of the $\beta$-arrestin- 2 expression and its binding ability with $\alpha$ l adrenergic receptor, thereby affecting the agonist-induced ROCK activation and improving the contractile response in blood vessels.
\end{abstract}

Key words: Reactive oxygen species; hypocontractility; mesenteric artery; norepinephrine; portal hypertension.

\section{Introduction}

Hyperdynamic circulation associated complications such as esophageal variceal hemorrhage is one of the important reasons for the deaths in patients with cirrhosis and portal hypertension [1]. Vasodilation resulted from hypo-contraction of arteries to vasoconstrictors plays a key role for the formation of 
portal hypertension and hyperdynamic circulation, which is involved in the increased affinity between $G$ protein-coupled receptors (GPCRs) and the $\beta$-arrestin-2, strengthening the receptor desensitization and affecting Rho associated coiled-coil forming protein kinase (ROCK) in RhoA/ROCK pathway [2-4].

Sympathetic nervous system excitation will stimulate the generation of reactive oxygen species (ROS) in blood circulation upon the formation of cirrhosis with portal hypertension. In addition, the high concentrations of angiotensin II in the plasma can activate the production of vascular endothelial cell membrane-associated NAD (P) $\mathrm{H}$ oxidase and superoxide anion $\left(\mathrm{O}_{2}-\right)$, resulting in oxidative stress in visceral and peripheral blood vessels $[5,6]$. ROS is involved in many biological processed such as ligand-receptor interaction, activation of transcription factors and gene expression. Therefore, the intracellular ROS, especially hydrogen peroxide $\left(\mathrm{H}_{2} \mathrm{O}_{2}\right)$, are considered to be new second messengers, which affect $\mathrm{Ca}^{2+}$ signaling pathway and the contractile function of vascular smooth muscle cells [7]. The $\mathrm{O}_{2}{ }^{-}$only functioned as an intracellular signaling molecule due to its difficulty of diffusion across cell membranes, whereas $\mathrm{H}_{2} \mathrm{O}_{2}$ both functions in intra/extracellular signal transduction pathway.

The shearing force to the vascular wall is increased due to the hyperdynamic circulation status in cirrhosis, resulting in increased ROS level in the vascular wall. N-acetylcysteine inhibits ROS production and effectively alleviates the hyperdynamic circulation in cirrhotic rats [8]. Vitamin C significantly decreases the ROS level and improves the hyporeactivity of forearm artery to vasoconstrictors such as norepinephrine (NE) and AT-II in patients with liver cirrhosis Child-Pugh grade C [9]. Losartan or apocynin reduces ROS content and ameliorates the low responses of blood vessel to vasoconstrictor in the mesenteric artery in rats induced by bile duct ligation [10]. Recent studies have reported that ROS can affect the function of ROCK in vascular smooth muscles in vascular diseases such as pulmonary hypertension [11], hypertension $[12,13]$ and cerebrovascular disease [14]. Therefore, we speculate that ROS may be participated in the regulation of ROCK in vascular smooth muscle.

In the present study, the contractile response of mesenteric artery to NE was determined before/after reducing the $\mathrm{ROS}$ levels in $\mathrm{CCl}_{4}$-induced cirrhotic rats with portal hypertension. Our research aimed to explore the roles of ROS in mesenteric artery contractility, GPCRs desensitization and RhoA/ROCK pathway in cirrhotic rats.

\section{Materials and Methods}

\section{Materials}

Healthy male Sprague-Dawley rats weighing 120 to $130 \mathrm{~g}$ were provided by Experimental Animal Center of School of Medicine, Shanghai Jiaotong University. The following antibodies were used in the study: Rabbit anti-ROCK-1 antibody (CST, American), normal rabbit IgG (sc-2027), rabbit anti-mosein antibody (sc-6410), rabbit anti-phospho-moesin threonine 555 (sc-12895), mouse anti- $\beta$-arrestin- 2 antibody (sc-6387), rabbit anti-a1 adrenergic receptor antibody. These antibodies were purchased from Santa Cruz, American. $\mathrm{H}_{2} \mathrm{O}_{2}$ detection kit and dihydroethidium (DHE) was purchased from Merck, Germany. Apocynin, tempol, PEG-catalase and Y27632 were purchased from Sigma, American.

\section{Experimental animal preparation and grouping}

Establishment of animal model was conducted according to the previous study [4]. To be brief, $60 \%$ $\mathrm{CCl}_{4}$ oil solution was injected intramuscularly accorded with dose volume $0.4 \mathrm{ml} / 100 \mathrm{~g}$ body weight, 2 times a week while drinking $5 \%$ ethanol. Within 14 to 16 weeks, this procedure led to the rat liver cirrhotic nodules with portal hypertension. When rats presented ascites, the exposure to $\mathrm{CCl}_{4}$ and alcohol was stopped for 7 days, and experiments were performed subsequently. Age-matched but untreated rats served as noncirrhotic controls.

Animals were randomly divided into groups. Rats with cirrhosis and portal hypertension were divided into four groups, i.e., PHTA (portal hypertension rats treated with apocynin), PHTT (portal hypertension rats treated with tempol), PHTP (portal hypertension rats treated with PEG-catalase) and PHTC (portal hypertension rats with vehicle). Normal rats were also divided into four groups, i.e., NA (normal rats treated with apocynin), NT (normal rats treated with tempol), NP (normal rats treated with PEG-catalase) and NC (normal rats treated with vehicle).

Apocynin $(30 \mathrm{mg} / \mathrm{kg} / \mathrm{d})$, tempol $(30 \mathrm{mg} / \mathrm{kg} / \mathrm{d})$ and PEG-catalase $(10000 \mathrm{u} / \mathrm{kg} / \mathrm{d})$ were dissolved in saline solution, filter sterilized with syringe filter and injected intraperitoneally. The control group was injected with saline solution. All rats were injected intraperitoneally once a day for 14 consecutive days. Based on previous studies, the injection dose and administration time of anti-oxidants were sufficient to reduce the levels of oxidative stress in the tested rats [15-18]. 


\section{Sampling and stimulation}

Rats were anesthetized with ketamine through intramuscular injection $(250 \mathrm{mg} / \mathrm{kg}$ ) and then fixed. Cut the abdomen in the midline and remove the mesenteric artery, as well as the connected mesentery and intestine, and divide the specimens into two groups. For the first group, we preserved them in 3-(N-morpholino) propanesulfonic acid (MOPS)-buffered physiological salt solution (MOPS-PSS) $\left(0 \sim 4{ }^{\circ} \mathrm{C}\right.$; $\left.\mathrm{pH} 7.4\right)$ that was composed of $\mathrm{NaCl}(145 \mathrm{mmol} / \mathrm{L}), \mathrm{KCl}(5.0 \mathrm{mmol} / \mathrm{L}), \mathrm{CaCl}_{2}(2.0$ $\mathrm{mmol} / \mathrm{L}), \quad \mathrm{MgSO}_{4} \quad(1.0 \mathrm{mmol} / \mathrm{L}), \quad \mathrm{NaH}_{2} \mathrm{PO}_{4} \quad(1.0$ $\mathrm{mmol} / \mathrm{L})$, dextrose $(5.0 \mathrm{mmol} / \mathrm{L})$, pyruvate $(2.0$ $\mathrm{mmol} / \mathrm{L})$, EDTA $(0.02 \mathrm{mmol} / \mathrm{L})$, and MOPS (3.0 $\mathrm{mmol} / \mathrm{L}$ ) [19]. These specimens were used to dissecting the third-order arterioles. For the second group, all of the mesenteric arteries were drawn out and frozen in liquid nitrogen immediately or transferred to MOPS-PSS and incubated at $37^{\circ} \mathrm{C}$ for $30 \mathrm{~min}$. NE was then added to the MOPS-PSS at a final concentration of $10 \mu \mathrm{M}$. The samples were incubated for another $20 \mathrm{~min}$ and then stored in liquid nitrogen for later use. Alternatively, the specimens were incubated in MOPS-PSS for $20 \mathrm{~min}$ at $37^{\circ} \mathrm{C}$ without stimulation by $\mathrm{NE}$ and then frozen in liquid nitrogen.

\section{ROS measurement with Dihydroethidium (DHE)}

Dihydroethidium oxidative fluorescence dye was used to evaluate the in situ production of ROS, as previously described [20]. Mesenteric artery was cut into slices with $10 \mu \mathrm{m}$ in thickness by means of a Frozen Slicer. DHE probe was dissolved with DMSO (dimethyl sulphoxide, DMSO), diluted with MOPS-PSS to a final concentration of $10 \mu \mathrm{mol} / \mathrm{L}$. $100 \mu \mathrm{l}$ of DHE probe solution was added dropwise to the slice surface, and immediately placed in the dark moisturizing box at $37^{\circ} \mathrm{C}$ for $30 \mathrm{~min}$. The sections were placed under a fluorescence microscope (Olympus, Japan) and observed with $535 \mathrm{~nm}$ wavelength. The images were collected using software for analysis.

\section{Measurement of $\mathrm{H}_{2} \mathrm{O}_{2}$}

Divalent iron ions were oxidized to ferric ions with $\mathrm{H}_{2} \mathrm{O}_{2}$ and formed a purple product with xylenol orange, which can be used for the detection of $\mathrm{H}_{2} \mathrm{O}_{2}$. To be brief, $10 \mathrm{mg}$ mesenteric artery tissue was homogenated, centrifugated at $4^{\circ} \mathrm{C}$ with $12000 \mathrm{~g}$ for 5 minutes, and the supernatant was used for subsequent experiment. All experiments were operated on ice. Finally, the test tubes containing $50 \mu 1$ of supernatants and $100 \mu \mathrm{l}$ of test solutions were placed at room temperature for $20 \mathrm{~min}$ and measured instantly with a spectrometer (Pharmacia, Sweden) at a wavelength of $560 \mathrm{~nm}$. The concentration of $\mathrm{H}_{2} \mathrm{O}_{2}$ released was cal- culated according to standard concentration curve originated from standard solutions upon the identical experiments.

\section{Determination the reactivity of mesenteric arteriole to NE}

With a dissecting microscope, the third-level of arteriole in mesentery was sharply dissected and transferred to a vascular perfusion system containing MOPS-PSS $\left(0 \sim 4^{\circ} \mathrm{C}, \mathrm{pH}=7.4\right)$. Glass micropipette

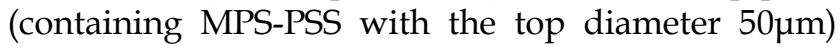
was inserted to one end of arteriole and fixed with 11-0 single-strands. Blood were flushed out with the perfusion pressure of $8 \mathrm{mmHg}$ and keep the other end of blood vessels open. Then insert another glass micropipette to the other end of the lumen and fix it with 11-0 line.

The vascular diameter was measured with microscope camera system and displayed on computer screen. The diameter change was determined by arteriole imaging feet and data were stored in computer. The diameter of blood vessels under the pressure of $80 \mathrm{mmHg}$ for $30 \mathrm{~min}$ was recorded as that of the basic state. According to the cumulative concentration method, NE was added to the vascular tank with the concentration from $10^{-7}$ to $10^{-5} \mathrm{M}$ and followed by recording the contraction rate of blood vessels under different concentrations. Cumulative dose- response curve for NE was drawn on the basis of contraction rate, i.e., the ratio of intravascular diameter change to maximum diameter. Vasoconstriction rate and the logarithm of NE concentration were used as vertical axis and abscissa, respectively. Compare the difference of curves among groups and record the NE concentration when the contraction rate is $50 \%$ (i.e., $\mathrm{EC}_{50}$ ).

To further investigate the role of ROCK in the vascular smooth muscle contractility of mesenteric artery affected by ROS, the effect of Y-27632 (a ROCK specific inhibitor), was observed. The above eight groups were divided into two subgroups: Y-27632 was added to one subgroup with a final concentration of $1 \mu \mathrm{M}$ in MOPS-PSS for 30min, referred to (Y-27632 +) group; another subgroup was added with the same amount of MOPS-PSS, referred to (Y-27632-) group [2]. Then record the vascular contractility stimulated with different concentrations of NE $\left(5 \times 10^{-7} \mathrm{M}\right.$, $1 \times 10^{-6} \mathrm{M}, 5 \times 10^{-6} \mathrm{M}$ and $\left.1 \times 10^{-5} \mathrm{M}\right)$. To reduce experimental error, two arterioles were taken from the same position and the reactivity to NE was measured under the same condition.

\section{Western blot}

The expression levels of the following proteins were detected by western blot: a1 adrenergic receptors, $\beta$-arrestin- 2 and ROCK- 1 , as well as moesin and 
phosphorylation of moesin (p-moesin). Activity of ROCK was assessed via the phosphorylation state of the ROCK substrate moesin at threonine 558 [21,22]. Using $\beta$-actin as an internal reference, the protein quantitative analysis was calculated by digital imaging software (Kodak, American).

\section{Co-immunoprecipitation}

Frozen mesenteric artery was thawed, homogenated in $1 \mathrm{ml}$ cell lysate, and centrifuged at $10000 \mathrm{~g}$ for $15 \mathrm{~min}$. The concentration of protein extracts was measured by the BCA protein Assay Kit (Pierce, USA). Protein extracts were incubated with normal rabbit IgG (50ul) in an ice bath for $1 \mathrm{~h}$, precleared with proteinA-agarose beads (Bio-Rad, Germany) at $4^{\circ} \mathrm{C}$ for $30 \mathrm{~min}$ and centrifuged at $12000 \mathrm{~g}$ for $10 \mathrm{~min}$ at $4^{\circ} \mathrm{C}$. Then the $80 \mu \mathrm{l}$ of the supernatant (about $50 \mu \mathrm{g}$ protein) were incubated with a1 adrenergic receptor antibody at $4^{\circ} \mathrm{C}$ overnight, washed in lysis buffer and centrifugated at $1000 \mathrm{~g}$ for $5 \mathrm{~min}$ at $4^{\circ} \mathrm{C}$. The precipitates were then stored in $-80^{\circ} \mathrm{C}$ or detected by Western blot with the $\beta$-arrestin- 2 antibody.

\section{Statistical analysis}

Data were given as the mean \pm SEM and analyzed by ANOVA for multiple comparisons with SPSS 16.0. A $P$ value $<0.05$ was considered statistically significant. The change in the reactivity of the mesenteric arteriole in response to NE was presented as a dose-response curve, which was fitted by nonlinear regression analysis (Graph Pad Software Inc., San Diego, CA., USA), and $\mathrm{EC}_{50}$ values were calculated from the fitted curve.

\section{Results}

\section{DHE fluorescent staining}

The fluorescence intensity of vascular wall was significantly increased in cirrhotic rats $(3.26 \pm 1.02$ vs 11.8 $\pm 2.19 \mathrm{AU}, \mathrm{P}<0.01)$. However, the fluorescence intensity was remarkably decreased in mesenteric artery after treatment with apocynin $(11.8 \pm 2.19$ vs $4.02 \pm 1.00 \mathrm{AU}, \mathrm{P}<0.01)$, tempol $(11.8 \pm 2.19$ vs $4.58 \pm 1.32$ $\mathrm{AU}, \mathrm{P}<0.01)$ or $\mathrm{PEG}$-catalase $(11.8 \pm 2.19$ vs $4.24 \pm 1.70$ AU, $\mathrm{P}<0.01$ ) (Fig. 1).

\section{$\mathrm{H}_{2} \mathrm{O}_{2}$ production in mesenteric artery}

In cirrhotic rats, the $\mathrm{H}_{2} \mathrm{O}_{2}$ content in mesenteric arterial wall was significantly higher compare to the normal control groups $(3.60 \pm 0.91$ vs $7.74 \pm 2.04 \mu \mathrm{M}$, $P<0.01)$. However, the $\mathrm{H}_{2} \mathrm{O}_{2}$ content was diminished by the pretreatment of apocynin $(7.74 \pm 2.04$ vs $4.04 \pm 1.27 \mu \mathrm{M}, P<0.01)$ or PEG-catalase $(7.74 \pm 2.04$ vs $3.83 \pm 1.40 \mu \mathrm{M}, P<0.01)$, but not by tempol treatment (7.74 \pm 2.04 vs $7.18 \pm 1.75 \mu \mathrm{M}, P>0.05$ ) (Fig. 2).

A

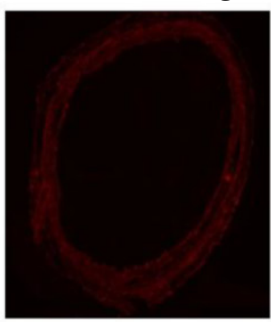

NC

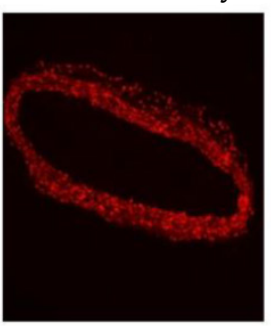

PHTC

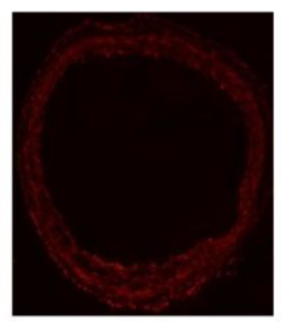

PHTA

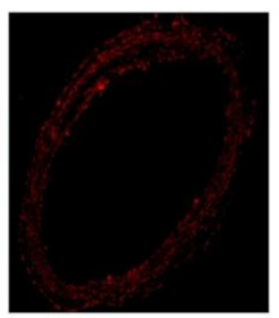

PHTT

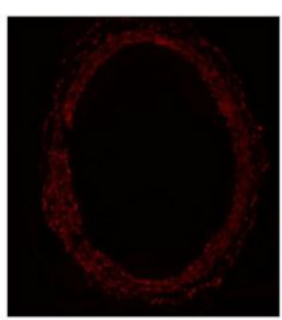

PHTP

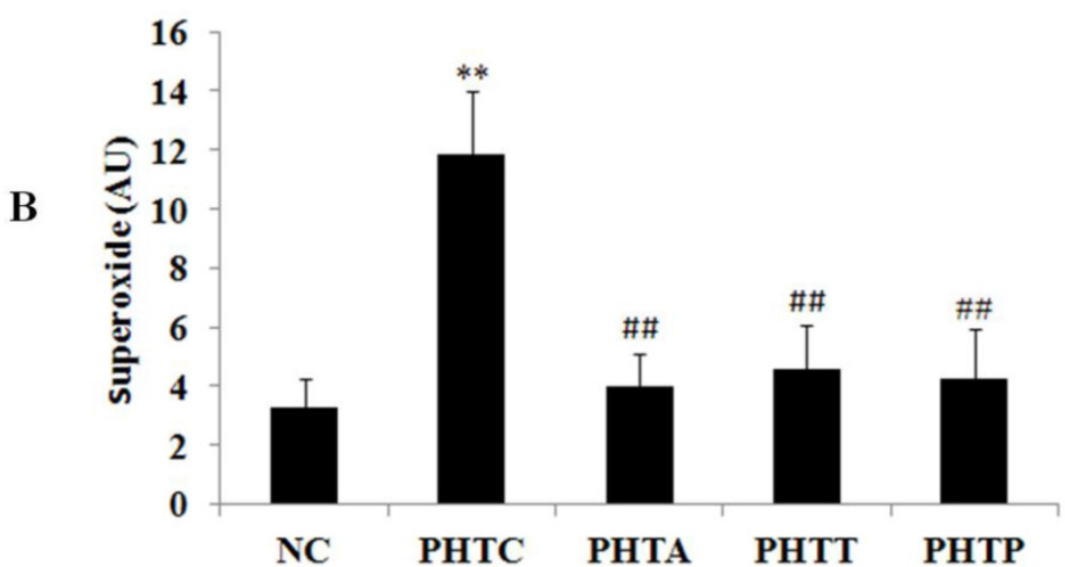

Figure I. Fluorescence intensity of vascular wall in rats after drugs treatment. (A) Fluorescence intensity was the strongest in cirrhotic rats and diminished by treatment with apocynin, tempol or PEG-catalase. Each image is representative of results from 6 different animals. (B) The quantitative of fluorescence intensity was analyzed in rats after treatment with different drugs. Values are mean \pm SEM; $n=6$ for each group. **: compared with NC, $P<0.01$; \#\#: compared with PHTC, $P<0.01$. 


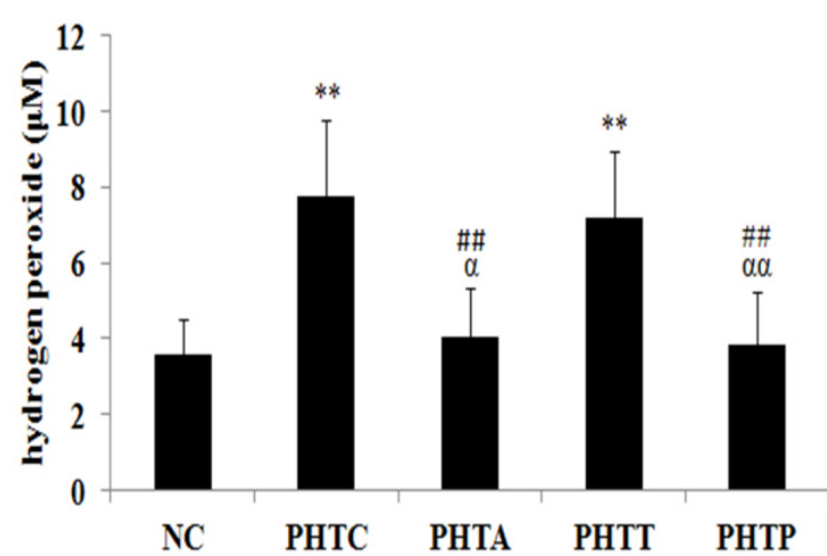

Figure 2. Determination of $\mathrm{H}_{2} \mathrm{O}_{2}$ content in rats after treatment with different drugs. $\mathrm{H}_{2} \mathrm{O}_{2}$ production in mesenteric artery was measured in cirrhotic rats after drugs treatment. Values are mean \pm SEM; $n=7$ for each group. **: compared with NC, $P<0.01$; \#\#: compared with PHTC, $P<0.01$; ad: compared with PHTT, $P<0.0$ I; $\alpha$ : compared with PHTT, $P<0.05$.

\section{Contraction response of mesenteric arteriole to NE}

With pretreatment of apocynin (Fig. 3A, 3B), the dose - response curves of mesenteric arteriole to $\mathrm{NE}$ moved to the left and the $\mathrm{EC}_{50}$ decreased in rats with cirrhosis. As shown in figure $3 \mathrm{C}$ and $3 \mathrm{D}$, similar results were also observed in cirrhotic rats after PEG-catalase treatment. However, such changes were not observed with the treatment of tempol (data not shown).

\section{Effect of $\mathrm{Y}-27632$ on the contraction response of mesenteric arterioles to NE}

To further investigate the contraction change under NE stimulation, the effect of ROCK inhibitor Y-27632 was determined by myographic experiments. As shown in Figure 4, Y-27632 inhibited NE-induced contraction of mesenteric arterioles in all groups. Meanwhile, the inhibition capacity was obviously decreased by pretreatment with apocynin or PEG-catalase, but not by tempol (Fig. 5).

$\mathbf{A}$
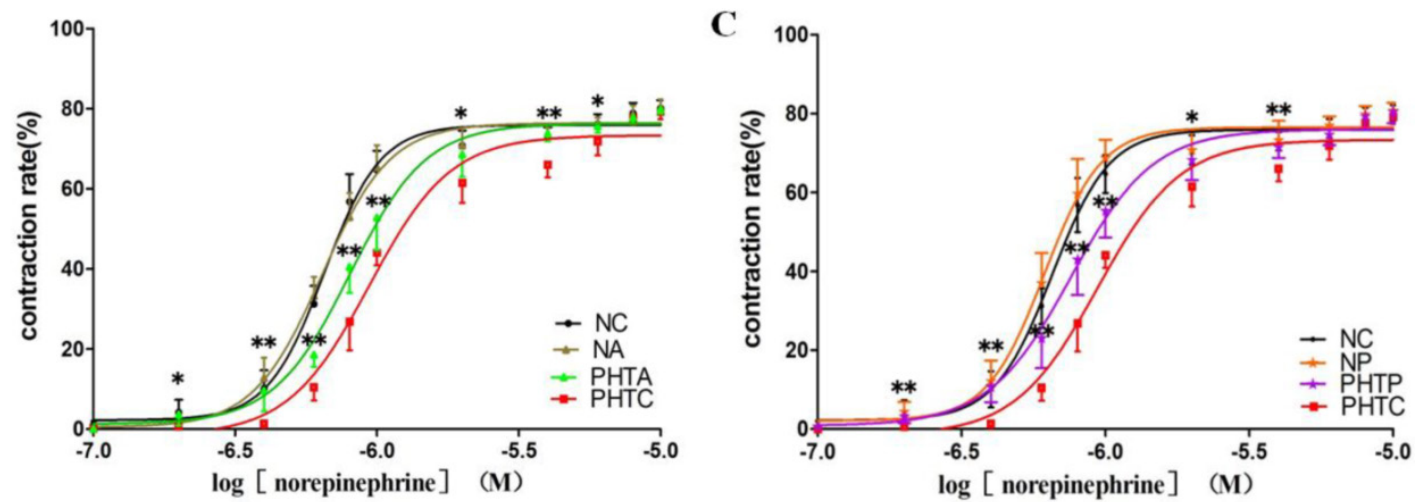

B $\quad \mathbf{E C 5 0}\left(\times 10^{-7} \mathbf{M}\right)$

D $\operatorname{EC50}\left(\times 10^{-7} \mathbf{M}\right)$

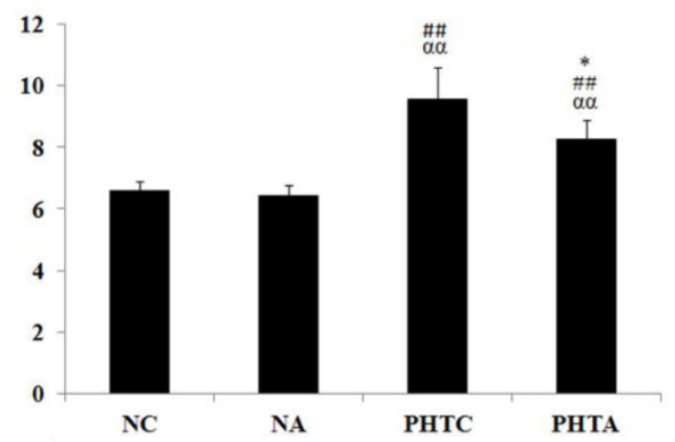

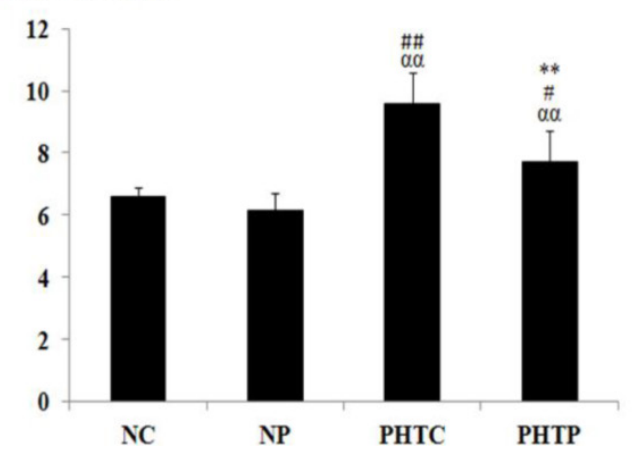

Figure 3. Dose-response curves of isolated mesenteric artery contractility to norepinephrine with different concentrations. Vasoconstriction rate and the logarithm of norepinephrine concentration were used as vertical axis and abscissa, respectively. The results showed that dose-response curve of mesenteric arteriole contractility to norepinephrine moved to the left and $\mathrm{EC}_{50}$ decreased in rats with cirrhosis in apocynin (A, B) or PEG-catalase (C, D) treatment. A: * PHTA compared with PHTC, P<0.05; ** PHTA compared with PHTC, P<0.0I. B: *: Compared with PHTC, P<0.05; \#\#: compared with NC, $P<0.0$ I; aq: compared with NA, $P<0.0$ I. C: * PHTP compared with PHTC, $P<0.05$; ** PHTP compared with PHTC, P<0.0I. D: **: Compared with PHTC, $P<0.0$ I; \#: compared with NC, $P<0.05$; \#\#: compared with NC, $P<0.0$ I; ad: compared with NP, $P<0.0$ I. 


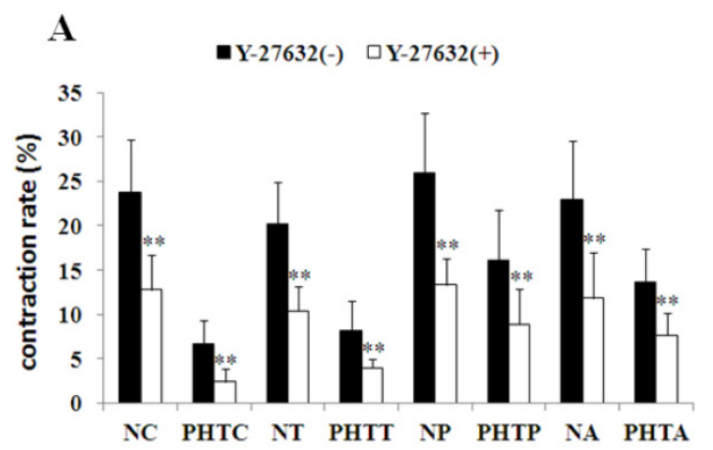

B

- Y-27632(-) वY-27632(+)
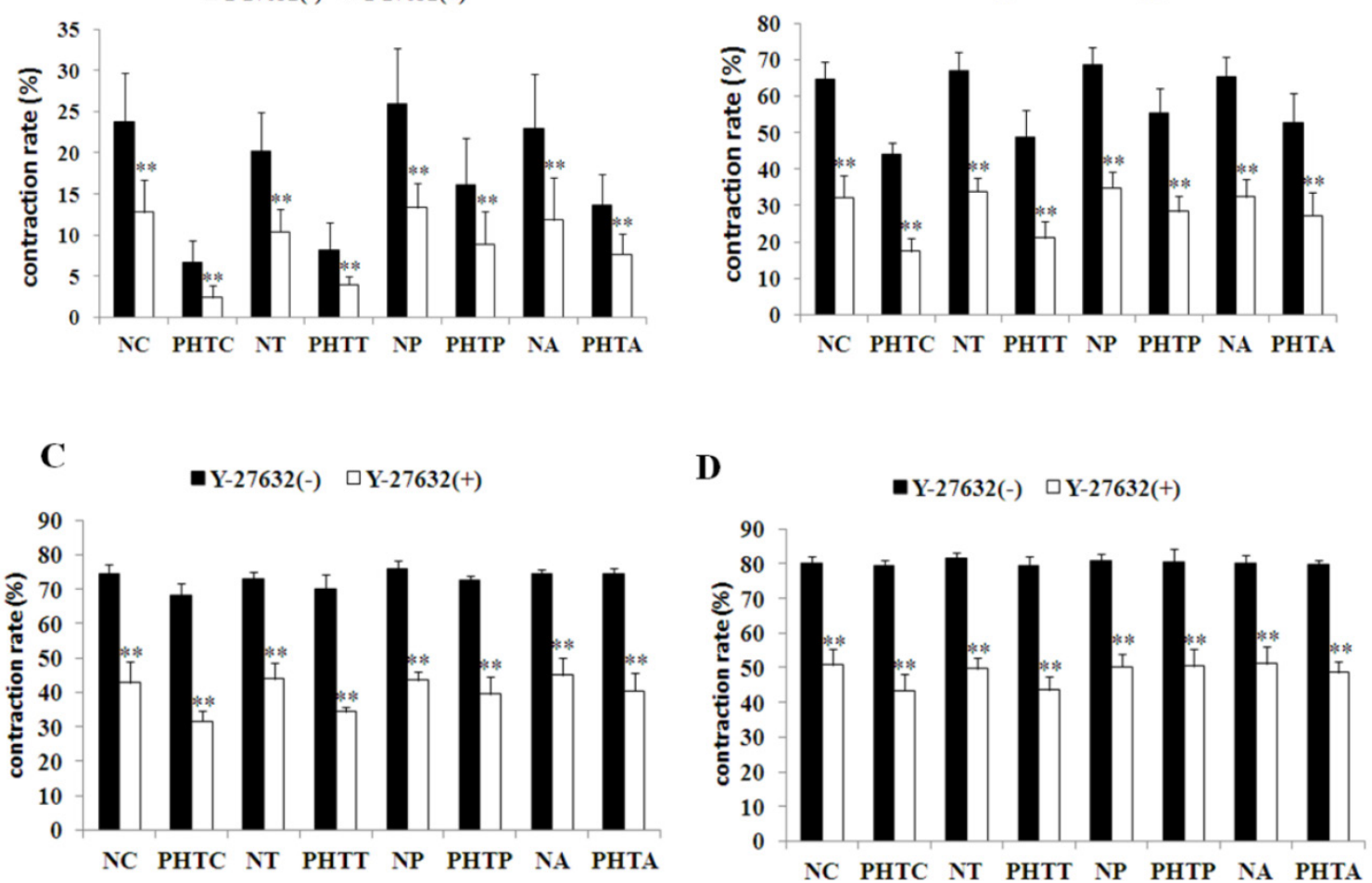

Figure 4. Contractility changes in different groups before and after incubation with Y-27632 upon norepinephrine-stimulation with different concentrations. Effect of the ROCK inhibitor Y-27632 on norepinephrine-induced contraction was assessed in myographic experiments after drugs treatment with different concentrations. $\left(A\right.$ : $5 \times 10^{-7} \mathrm{M}$; B: I $\times 10^{-6} \mathrm{M}$; C: $5 \times 10^{-6} \mathrm{M}$; D: $\left.1 \times 10^{-5} \mathrm{M}\right)$. Values are mean $\pm \mathrm{SEM}$; $\mathrm{n}=7$ for each group. $* *$ : Compared with the contractility before incubation with $Y-27632, P<0.01$

A

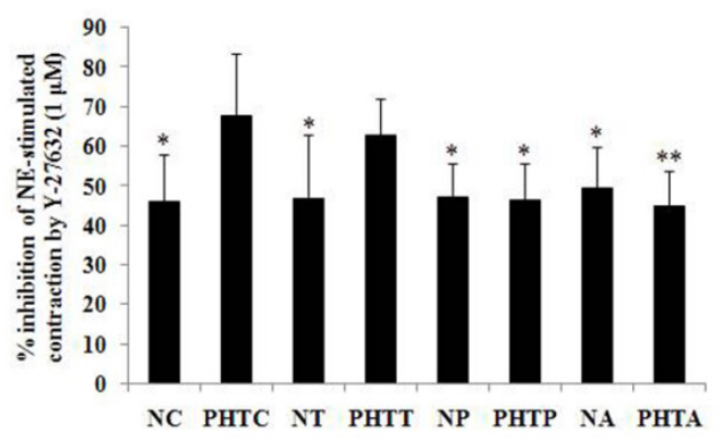

C

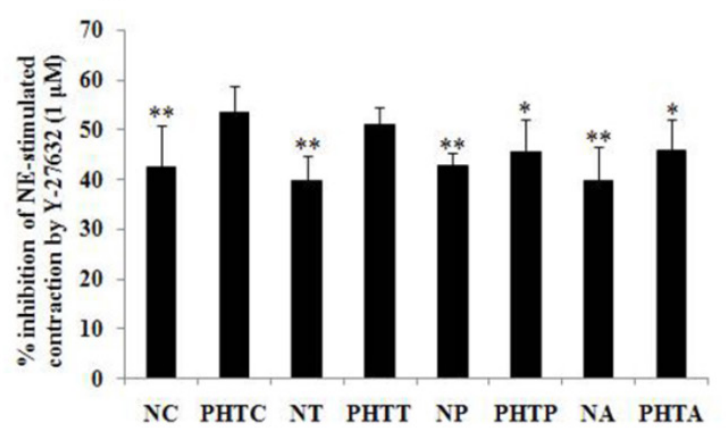

B

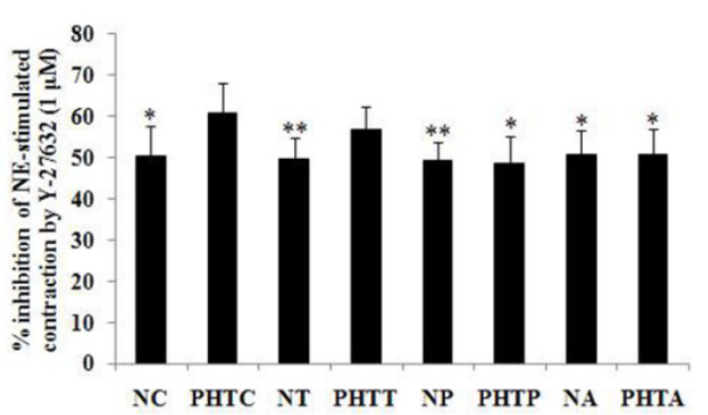

D

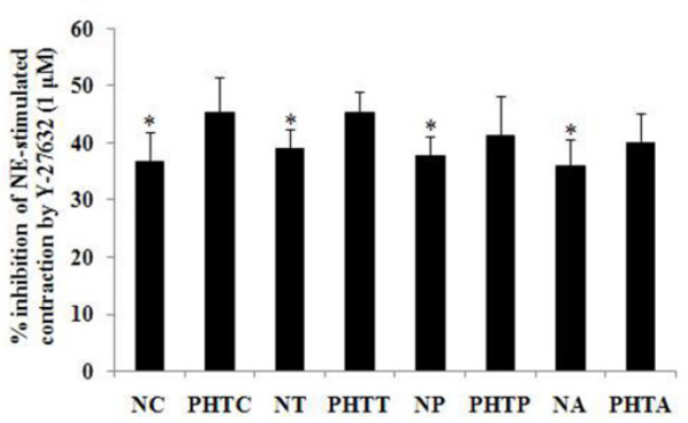

Figure 5. Inhibition effect of Y-27632 on the contraction response of mesenteric arterioles to NE after antioxidants treatment. Inhibition effect of the ROCK inhibitor $Y-27632$ on norepinephrine-induced contraction was assessed in myographic experiments after antioxidants treatment with different concentrations. $\left(\mathbf{A}: 5 \times 10^{-7} \mathrm{M}\right.$; B: I $\times 10^{-6} \mathrm{M} ; \mathbf{C}: 5 \times 10^{-6} \mathrm{M}$; D: $\left.1 \times 10^{-5} \mathrm{M}\right)$. Values are mean \pm SEM; $\mathrm{n}=7$ for each group. *: Compared with PHTC, P<0.05; **: compared with PHTC, $P<0.01$ 


\section{Expression and interaction of $\alpha \mathrm{I}$ adrenergic receptor and $\beta$-arrestin-2}

a1 adrenergic receptor and $\beta$-arrestin- 2 expression in all the groups was detected by western blot as shown in Figure 6A. As the result indicated, the expression of a1 adrenergic receptor showed no significant change with treatment of apocynin, tempol or PEG-catalase in normal or cirrhotic rats (data not shown). Meanwhile, $\beta$-arrestin-2 expression was significantly higher in cirrhotic rats, which was abolished by pretreatment with apocynin or PEG-catalase. Besides, there are no significant differences in all the non-cirrhotic rats (Fig. 6B). Furthermore, we investigated the interaction between $\alpha 1$ adrenergic receptor and $\beta$-arrestin- 2 using co-immunoprecipitation. The results showed that the adrenergic receptor co-immunoprecipitated with $\beta$-arrestin-2 and the binding affinity was higher in cirrhotic rats, which was diminished by administration with apocynin or PEG-catalase, but not by tempol (Fig. 6C).

\section{NE induces the expression and activation of ROCK-I}

We compared the protein expression of ROCK-1 between the NC group and cirrhotic rats upon stimulation with NE. The results showed that ROCK-1 expression both in normal and cirrhotic rats exhibited no significant changes ( $p>0.05)$. The same results were observed in all other groups (data not shown). Then we compared the activity of ROCK- 1 between the NC group and PHTC group upon stimulation with NE. The result showed that it is the phosphorylation level of moesin that significantly increased upon NE stimulation in NC group. Meanwhile, there are no obvious changes for moesin and p-moesin expression in PHTC group (Fig.7A). Similarly, such results were observed in NT and PHTT groups (Fig.7B). Furthermore, the phosphorylation of moesin was significantly increased in NP/PHTP and NA/PHTA groups upon NE stimulation (Fig. 7C and 7D).
A

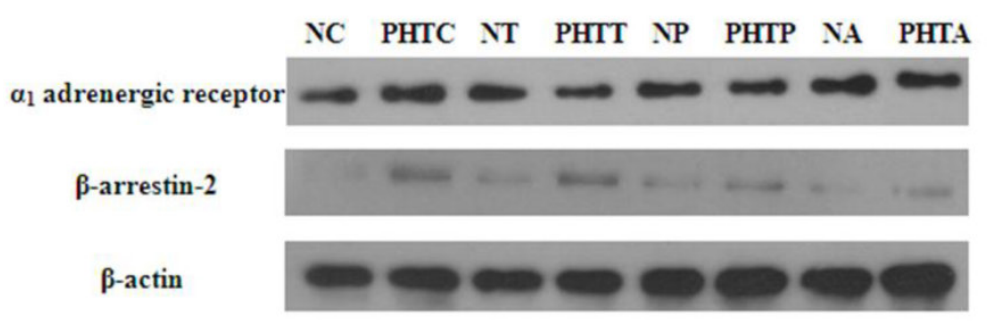

C

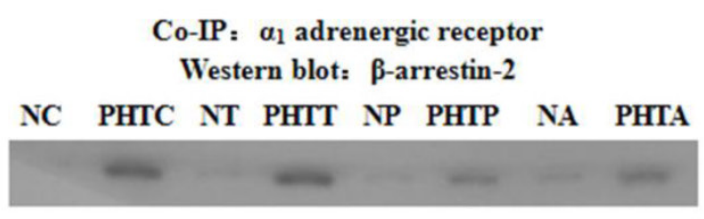

B
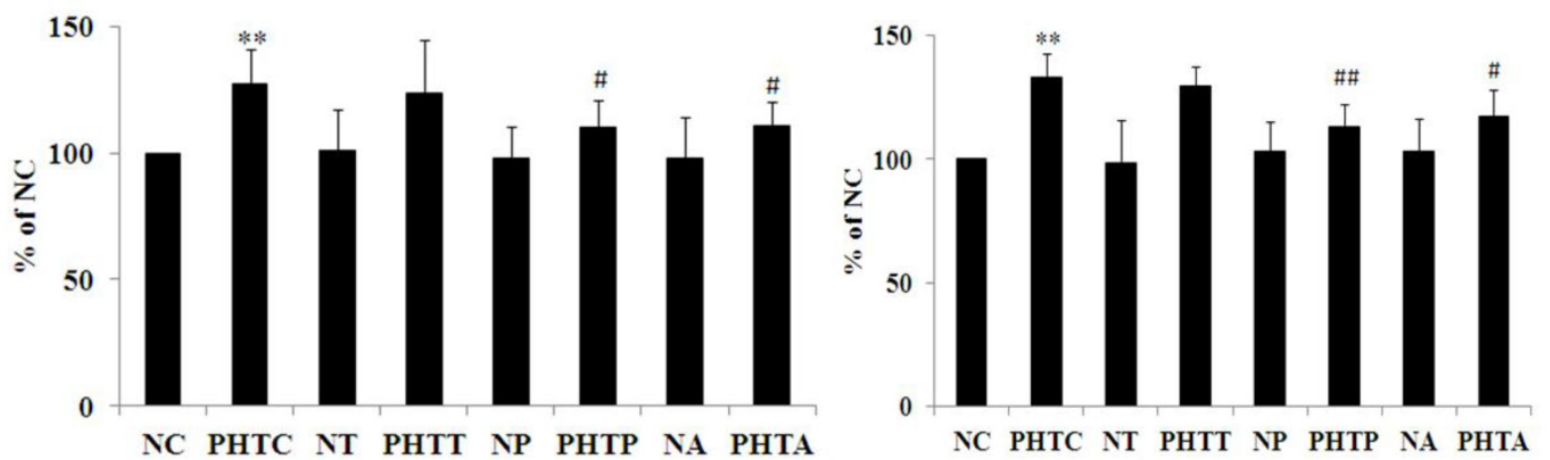

Figure 6. Expression and interaction of $\alpha \mathrm{I}$ adrenergic receptor and $\beta$-arrestin-2. Western blot was used to determine the expression of $\alpha \mathrm{I}$ adrenergic receptor and $\beta$-arrestin-2. Co-immunoprecipitation was performed to investigate the interaction between $\alpha \mathrm{I}$ adrenergic receptor and $\beta$-arrestin-2. $(\mathbf{A}) \alpha \mathbf{I}$ adrenergic receptor and $\beta$-arrestin-2 expression in all the groups. (B) Quantitative analysis of $\beta$-arrestin- 2 expression. The bar graphs show the relative expression levels of indicated proteins after normalization to $\beta$-actin. Values are mean \pm SEM; $n=7$ for each group. (C) Interaction between the $\alpha$ I adrenergic receptor and $\beta$-arrestin-2 in mesenteric artery of the rats. $\mathrm{n}=7$ for each group. $* *$ : compared with $\mathrm{NC}, \mathrm{P}<0.0 \mathrm{I}$; \#: compared with $\mathrm{PHTC}$, $P<0.05$; \#\#: compared with PHTC, $P<0.0$ I. 
A

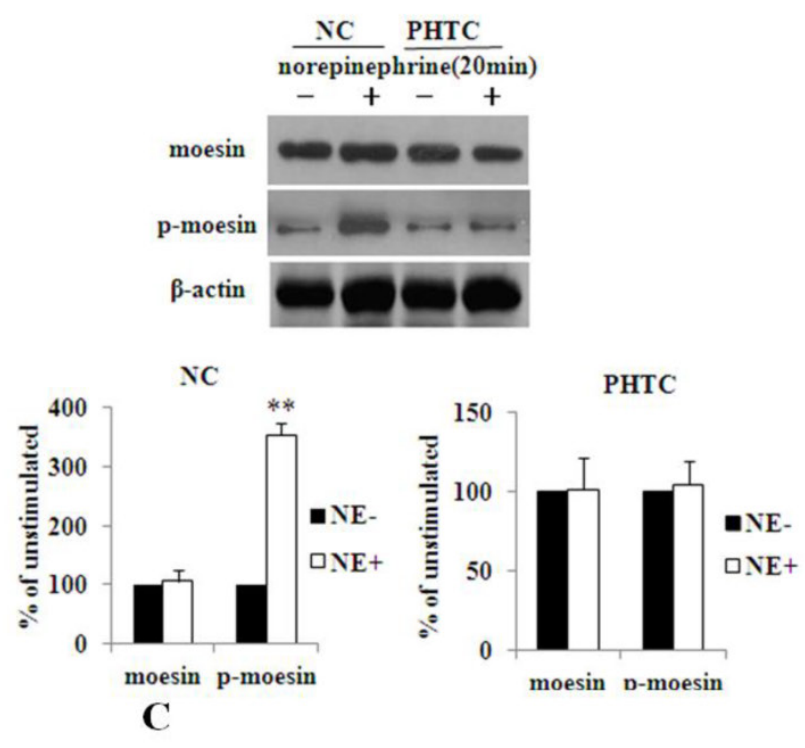

B

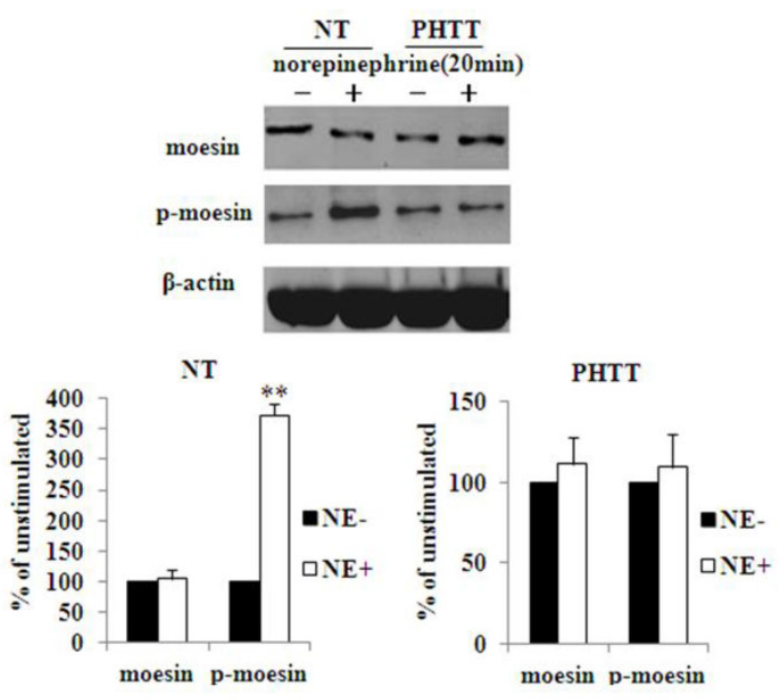

D
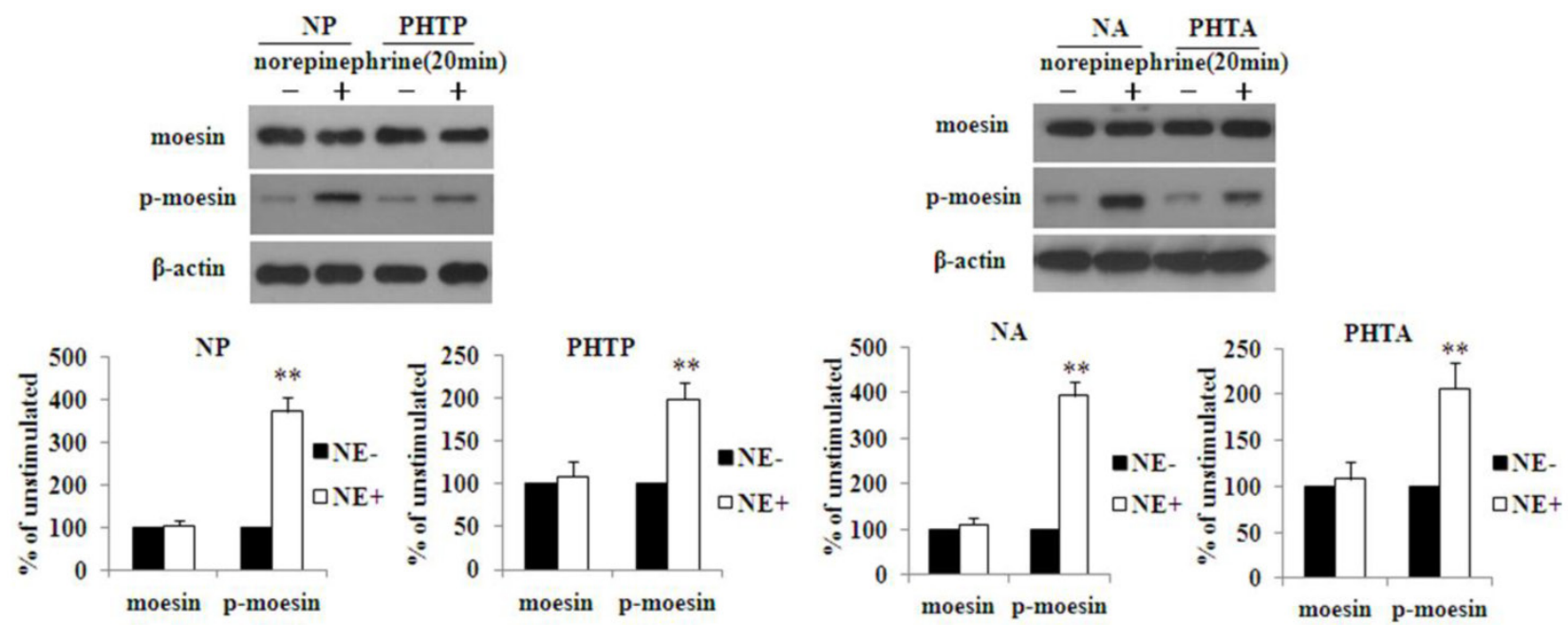

Figure 7. Change in activity of ROCK-I between the normal groups and cirrhotic groups upon NE stimulation. Western blot was used to measure the protein expression of moesin and p-moesin in different groups upon stimulation with NE. A: NC and PHTC group; B: NT and PHTT group; C: NP and PHTP group; D: NA and PHTA group. The bar graphs show the relative expression levels of indicated proteins after normalization to $\beta$-actin. The levels of moesin and $p$-moesin found in mesenteric arteries from rats without norepinephrine stimulation were set to I00\%. Values are mean \pm SEM; $n=6$ for each group. **: $P<0.01$, compared with the group without norepinephrine stimulation. NE: norepinephrine.

\section{Discussion}

The potential sources of ROS include mitochondrial respiratory chain, nitric oxide synthase, NAD(P) $\mathrm{H}$ oxidase and xanthine oxidase $(\mathrm{XO})$-catalyzed reactions [23]. Among these, NAD (P) H oxidase is generally considered the major source of vascular ROS and has been shown to increase the ROS production in the vascular tissue [24,25]. A variety of factors, such as virulence agents, cytokines, shear force and ischemia, can activate $\mathrm{NAD}(\mathrm{P}) \mathrm{H}$ oxidase $[26,27]$. Apocynin is a $\mathrm{NAD}(\mathrm{P}) \mathrm{H}$ oxidase specific inhibitor and effectively reduces the ROS production. A study found that apocynin could inhibit the generation of $\mathrm{O}_{2} \cdot{ }^{-}$, im- prove the mesenteric artery endothelial function and restore visceral blood flow in cirrhotic rats [10]. DHE fluorescence staining showed that the $\mathrm{O}_{2}{ }^{-}$content in mesenteric artery from cirrhotic rat treated with apocynin significantly decreased, indicating that apocynin inhibited $\mathrm{NAD}(\mathrm{P}) \mathrm{H}$ oxidase activity and reducing ROS generation. Simultaneously, in apocynin treated cirrhotic rat, the dose- response curve of mesenteric arteriole to NE moved to the left and the $\mathrm{EC}_{50}$ decreased, indicating the increased sensitivity of mesenteric artery to NE.

Mesenteric artery ROS in cirrhotic rats, especially $\mathrm{O}_{2}{ }^{-}$, exhibit a significantly higher intracellular concentration [28-30]. Meanwhile, superoxide dis- 
mutase (SOD) cannot penetrate the cell membrane and reduces intracellular $\mathrm{O}_{2}{ }^{-}$level [18]. Tempol, a superoxide dismutase mimic, can freely enter the cell, react and degrade with $\mathrm{O}_{2} \cdot{ }^{-}$subsequently, thus protecting cells from oxidative damage [31]. A recent study reported that recombinant human manganese SOD treatment significantly reduced portal pressure in cirrhotic rats, but failed to improve extrahepatic visceral arteries hyperdynamic circulation [32]. In accordance with this study, the dose response curves and $\mathrm{EC}_{50}$ showed no obvious change with treatment of tempol, indicating that tempol has no influence on the contractile response of mesenteric arteries to NE. Previous report showed that tempol increases nitric oxide and reduces portal pressure in sinusoidal endothelial cells and in cirrhotic livers [33]. However, contrary to the intrahepatic situation, tempol or SOD failed to improve the hypocontraction and hyperdynamic circulation due to the abundance of nitric oxide and hemangiectasis of extrahepatic vessels in portal hypertension. SOD and tempol can disproportionate $\mathrm{O}_{2} \cdot{ }^{-}$, but can not decompose its product $\mathrm{H}_{2} \mathrm{O}_{2}$, resulting the increased concentration of $\mathrm{H}_{2} \mathrm{O}_{2}$ in the cell. SOD plays an important role in the synthesis of endogenous $\mathrm{H}_{2} \mathrm{O}_{2}$ that contributes to reactive hyperemia in mouse mesenteric smaller arterioles[34]. Our study demonstrated that the content of $\mathrm{H}_{2} \mathrm{O}_{2}$ was increased in mesenteric artery of cirrhotic rats and induced the hypocontractility, which is reversed by the down-regulation of $\mathrm{H}_{2} \mathrm{O}_{2}$. Previous study showed that $\mathrm{H}_{2} \mathrm{O}_{2}$ induced eNOS gene expression and $\mathrm{NO}$ synthesis in aortic endothelial cells, which was involved in the activation of $\mathrm{Ca}^{2+} /$ calmodulin-dependent protein kinase II and Janus kinase $2[35,36]$. Cai et al found that endogenous $\mathrm{H}_{2} \mathrm{O}_{2}$, derived from the NAD $(\mathrm{P}) \mathrm{H}$ oxidase, mediated $\mathrm{NO}$ production in response to angiotensin II in normal endothelial cells, which could be reversed by catalase [37]. The content of angiotensin II is increased in cirrhotic rats and reducing the generation of $\mathrm{H}_{2} \mathrm{O}_{2}$ improves the visceral artery hyporesponsiveness, indicating the involvement of $\mathrm{H}_{2} \mathrm{O}_{2}$ in artery response to vasoconstrictors. This is consistent with our resutls and the mechanism may be invovled in the $\mathrm{K}^{+}$channel activation by excessive intravascular $\mathrm{H}_{2} \mathrm{O}_{2}$ [38-41]. The expression of $\beta$-arrestin- 2 and its binding ability with GPCRs increased in cirrhotic rats, which was diminished by pretreatment of apocynin or PEG-catalase, suggesting that apocynin or PEG-catalase can decrease the $\mathrm{H}_{2} \mathrm{O}_{2}$ content and attenuate the signal transduction inhibition of a1 adrenergic receptor by $\beta$-arrestin- 2 .

Inhibition of intracellular ROS also can abrogate both the $\beta$-arrestin and $G$ protein-mediated phosphorylation of ERK1/2 upon agonism of $\beta 2$-adrenergic receptor [42], but the interaction of ROS and $\beta$-arrestin-2 has not been elucidated. Zhang et al [43] has reported that $\beta$-arrestin plays a negative regulatory role in $\mathrm{H}_{2} \mathrm{O}_{2}$-induced apoptosis signaling through associating with apoptosis signal-regulating kinase and facilitating its degradation, which protects cells from oxidative stress-induced apoptosis. $\beta$-arrestin-2 also can protect cell death from an excessive oxidative burst[44]. Knock out of $\beta$-arrestin- 2 in mice led to the increase of $\mathrm{O}_{2} \cdot{ }^{-}$content and myeloperoxidase activity $[45,46]$, suggesting the negative regulation of $\beta$-arrestin-2 in oxidative stress. Therefore, the $\mathrm{O}_{2}{ }^{-}$and $\mathrm{H}_{2} \mathrm{O}_{2}$ levels were increased in mesenteric artery in cirrhotic rats. $\beta$-arrestin- 2 is an adaption protein and its expression is increased upon $\mathrm{O}_{2}$. and $\mathrm{H}_{2} \mathrm{O}_{2}$ stimulation.

Studies [47] have shown that ROCK is a critical mediator of vasocontraction. In the present research, we found that, in both normal and cirrhotic rats, there are no significant differences with respect to the ROCK and moesin expression, but the p-moesin has changed, suggesting that NE stimulation has an obvious effect on ROCK activity measured by its substrate moesin phosphorylation at threonine 558 .

The $\mathrm{p}$-moesin was increased in cirrhotic rat upon stimulation by apocynin or PEG-catalase, not by tempol. This result suggested that the increased sensitivity to NE was resulted from ROCK activation (not from its expression), the mechanism of which may be involved in reducing $\beta$-arrestin- 2 expression and its interaction with $\alpha 1$ adrenergic receptor and releasing $\mathrm{G}$ protein-dependent signal pathway dysfunction.

In addition, Y-27632 is a specific ROCK inhibitor and significantly inhibited NE-induced contractile response, revealing the critical role of ROCK in mesenteric artery contraction. In the current study, the inhibition ability of Y-27632 to arteriole contraction was observed using NE stimulation with different concentrations. The result also showed that Y-27632 has an obvious inhibition effect on NE-induced contraction in cirrhotic rat which was diminished by pretreatment with apocynin or PEG-catalase, but not by tempol. The result showed that reduction of $\mathrm{H}_{2} \mathrm{O}_{2}$ in mesenteric artery weakened the activity of Y-27632 on blocking NE-activated ROCK.

In summary, our study demonstrated that decrease of $\mathrm{H}_{2} \mathrm{O}_{2}$ in mesenteric artery from rats with cirrhosis resulted in the down regulation of the $\beta$-arrestin-2 expression and its affinity with a1 adrenergic receptor, thereby affecting the agonist-induced ROCK activation and improving the contractile response in blood vessels.

\section{Acknowledgement}

We thank Associate Professor Dr. Dong Sun 
(Department of Physiology, New York Medical College, Valhalla, New York, USA) and Professor Chang-dong Yan (Department of Physiology, XuZhou Medical College, XuZhou, China) for technical support and providing us with the vessel perfusion system.

Funding: This study was supported by a grant from the National Natural Science Foundation of China (No. 30972920).

\section{Competing Interests}

The authors have declared that no competing interest exists.

\section{References}

1. Khaderi S, Barnes D. Preventing a first episode of esophageal variceal hemorrhage. Cleve Clin J Med 2008; 75: 235-244.

2. Hennenberg M, Trebicka J, Biecker E, et al. Vascular dysfunction in human and rat cirrhosis: role of receptor-desensitizing and calcium-sensitizing proteins. Hepatology 2007; 45: 495-506.

3. Hennenberg M, Trebicka J, Kohistani AZ, et al. Vascular hyporesponsiveness to angiotensin II in rats with $\mathrm{CCl}(4)$-induced liver cirrhosis. Eur J Clin Invest 2009; 39: 906-913.

4. Chen W, Sang JY, Liu DJ, et al. Desensitization of G-protein-coupled receptors induces vascular hypocontractility in response to norepinephrine in the mesenteric arteries of cirrhotic patients and rats. Hepatobiliary Pancreat Dis Int 2013; 12: 295-304

5. Heller J, Trebicka J, Shiozawa $\mathrm{T}$, et al. Vascular, hemodynamic and renal effects of low-dose losartan in rats with secondary biliary cirrhosis. Liver Int 2005; 25: 657-666.

6. MØller S, Bendtsen F, Henriksen JH. Determinants of the renin-angiotensin-aldosterone system in cirrhosis with special emphasis on the central blood volume. Scand J Gastroenterol 2006; 41: 451-458.

7. Touyz RM. Reactive oxygen species in vascular biology: role in arterial hypertension. Expert Rev Cardiovasc Ther 2003; 1: 91-106.

8. Fernando B, Marley R, Holt S, et al. N-acetylcysteine prevents development of the hyperdynamic circulation in the portal hypertensive rat. Hepatology 1998; 28: 689-694.

9. Ferlitsch A, Pleiner J, Mittermayer F, et al. Vasoconstrictor hyporeactivity can be reversed by antioxidants in patients with advanced alcoholic cirrhosis of the liver and ascites. Crit Care Med 2005; 33: 2028-2033.

10. Dal-Ros S, Oswald-Mammosser M, Pestrikova T, et al. Losartan prevents portal hypertension-induced, redox-mediated endothelial dysfunction in the mesenteric artery in rats. Gastroenterology 2010; 138: 1574-1584.

11. Jernigan NL, Walker BR, Resta TC. Reactive oxygen species mediate RhoA/Rho kinase-induced $\mathrm{Ca} 2+$ sensitization in pulmonary vascular smooth muscle following chronic hypoxia. Am J Physiol Lung Cell Mol Physiol 2008; 295: L515-L529.

12. Jackson EK, Gillespie DG, Zhu C, et al. Alpha2-adrenoceptors enhance angiotensin II-induced renal vasoconstriction: role for NADPH oxidase and RhoA. Hypertension 2008; 51: 719-726.

13. Tsai MH, Jiang MJ. Reactive oxygen species are involved in regulating alpha1-adrenoceptor-activated vascular smooth muscle contraction. J Biomed Sci 2010; 17: 67.

14. Miller AA, Budzyn K, Sobey CG. Vascular dysfunction in cerebrovascular disease: mechanisms and therapeutic intervention. Clin Sci (Lond) 2010; 119: $1-17$

15. Sousa T, Pinho D, Morato M, et al. Role of superoxide and hydrogen peroxide in hypertension induced by an antagonist of adenosine receptors. Eur J Pharmacol 2008; 588: 267-276.

16. Angermayr B, Fernandez $M$, Mejias $M$, et al. NAD $(\mathrm{P}) \mathrm{H}$ oxidase modulates angiogenesis and the development of portosystemic collaterals and splanchnic hyperaemia in portal hypertensive rats. Gut 2007; 56: 560-564.

17. Yuksel BC, Serdar SE, Tuncel A, et al. Effect of tempol, a membrane-permeable radical scavenger, on mesenteric blood flow and organ injury in a murine cecal ligation and puncture model of septic shock. Eur Surg Res 2009; 43: 219-227.

18. Cuzzocrea S, McDonald MC, Mazzon E, et al. Beneficial effects of tempol, a membrane-permeable radical scavenger, on the multiple organ failure induced by zymosan in the rat. Critl Care Med 2001; 29: 102-111.

19. Falcone JC, Kuo L, Meininger GA. Endothelial cell calcium increases during flow-induced dilation in isolated arterioles. Am J Physiol 1993; 264(2 Pt 2): H653-H659.

20. Ejiri $\mathrm{J}$, Inoue $\mathrm{N}$, Tsukube $\mathrm{T}$, et al. Oxidative stress in the pathogenesis of thoracic aortic aneurysm: protective role of statin and angiotensin II type 1 receptor blocker. Cardiovasc Res 2003; 59: 988-996.
21. Trebicka J, Leifeld L, Hennenberg M, et al. Hemodynamic effects of urotensin II and its specific receptor antagonist palosuran in cirrhotic rats. Hepatology 2008; 47: 1264-1276.

22. Zhou Q, Hennenberg M, Trebicka J, et al. Intrahepatic upregulation of RhoA and Rho-kinase signalling contributes to increased hepatic vascular resistance in rats with secondary biliary cirrhosis. Gut 2006; 55: 1296-1305.

23. Brandes RP, Kreuzer J. Vascular NADPH oxidases: molecular mechanisms of activation. Cardiovasc Res 2005; 65: 16-27.

24. De Keulenaer GW, Alexander RW, Ushio-Fukai M, et al. Tumour necrosis factor alpha activates a p22phox-based NADH oxidase in vascular smooth muscle. Biochem J 1998; 329: 653-657.

25. Li JM, Shah AM. Mechanism of endothelial cell NADPH oxidase activation by angiotensin II. Role of the p47phox subunit. J Biol Chem 2003; 278: 12094-12100.

26. Cai H, Griendling KK, Harrison DG. The vascular NAD $(\mathrm{P}) \mathrm{H}$ oxidases as therapeutic targets in cardiovascular diseases. Trends Pharmacolo Sci 2003; 24: 471-478

27. Grote K, Flach I, Luchtefeld M, et al. Mechanical stretch enhances mRNA expression and proenzyme release of matrix metalloproteinase-2 (MMP-2) via NAD(P)H oxidase-derived reactive oxygen species. Circ Res 2003; 92: e80-e86.

28. Vercelino R, Tieppo J, Dias AS, et al. N-acetylcysteine effects on genotoxic and oxidative stress parameters in cirrhotic rats with hepatopulmonary syndrome. Basic Clin Pharmacol Toxicol 2008; 102: 370-376.

29. Lee KC, Yang YY, Wang YW, et al. Increased plasma malondialdehyde in patients with viral cirrhosis and its relationships to plasma nitric oxide, endotoxin, and portal pressure. Dig Dis Sci 2010; 55: 2077-2085

30. Chen MF, Mo LR, Lin RC, et al. Increase of resting levels of superoxide anion in the whole blood of patients with decompensated liver cirrhosis. Free Radic Biol Med 1997; 23: 672-679.

31. Mitchell JB, Samuni A, Krishna MC, et al. Biologically active metal-independent superoxide dismutase mimics. Biochemistry 1990; 29: 2802-2807

32. Guillaume M, Rodriguez-Vilarrupla A, Gracia-Sancho J, et al. Recombinant human manganese superoxide dismutase reduces liver fibrosis and portal pressure in CCl4-cirrhotic rats. J Hepatol 2013; 58: 240-246.

33. Garcia-Caldero H, Rodriguez-Vilarrupla A, Gracia-Sancho J, et al. Tempol administration, a superoxide dismutase mimetic, reduces hepatic vascular resistance and portal pressure in cirrhotic rats. J Hepatol 2011; 54(4): 660-665.

34. Yada T, Shimokawa H, Morikawa K, et al. Role of $\mathrm{Cu}, \mathrm{Zn}$-SOD in the synthesis of endogenous vasodilator hydrogen peroxide during reactive hyperemia in mouse mesenteric microcirculation in vivo. Am J Physiol Heart Circ Physiol 2008; 294: H441-H448.

35. Drummond GR, Cai H, Davis ME, Ramasamy S, et al. Transcriptional and posttranscriptional regulation of endothelial nitric oxide synthase expression by hydrogen peroxide. Circulation Research 2000; 86: 347-354.

36. Cai H, Davis ME, Drummond GR, et al. Induction of endothelial NO synthase by hydrogen peroxide via a $\mathrm{Ca}(2+) /$ calmodulin-dependent protein kinase II/janus kinase 2-dependent pathway. Arterioscler Thromb Vasc Biol 2001; 21: 1571-1576.

37. Cai H, Li Z, Dikalov $\mathrm{S}$, et al. NAD $(\mathrm{P}) \mathrm{H}$ oxidase-derived hydrogen peroxide mediates endothelial nitric oxide production in response to angiotensin II. J Biol Chem 2002; 277: 48311-48317.

38. Moreau R, Komeichi H, Cailmail S, et al. Blockade of ATP-sensitive K+ channels by glibenclamide reduces portal pressure and hyperkinetic circulation in portal hypertensive rats. J Hepatol 1992; 16: 215-218.

39. Sobey CG, Heistad DD, Faraci FM. Mechanisms of bradykinin-induced cerebral vasodilatation in rats. Evidence that reactive oxygen species activate $\mathrm{K}+$ channels. Stroke 1997; 28: 2290-2295.

40. Thengchaisri N, Kuo L. Hydrogen peroxide induces endothelium-dependent and -independent coronary arteriolar dilation: role of cyclooxygenase and potassium channels. Am J Physiol Heart Circ Physiol 2003; 285: H2255-H2263.

41. Atucha NM, Ortiz MC, Fortepiani LA, et al. Role of cyclic guanosine monophosphate and $\mathrm{K}+$ channels as mediators of the mesenteric vascular hyporesponsiveness in portal hypertensive rats. Hepatology 1998; 27: 900-905.

42. Singh M, Moniri NH. Reactive oxygen species are required for beta2 adrenergic receptor-beta-arrestin interactions and signaling to ERK1/2. Biochem Pharmacol 2012; 84: 661-669.

43. Zhang Z, Hao J, Zhao Z, et al. beta-Arrestins facilitate ubiquitin-dependent degradation of apoptosis signal-regulating kinase 1 (ASK1) and attenuate H2O2-induced apoptosis. Cell Signal 2009; 21: 1195-1206.

44. Zhao M, Wimmer A, Trieu K, et al. Arrestin regulates MAPK activation and prevents NADPH oxidase-dependent death of cells expressing CXCR2. J Biol Chem 2004; 279: 49259-49267.

45. Su Y, Raghuwanshi SK, Yu Y, et al. Altered CXCR2 signaling in beta-arrestin-2-deficient mouse models. J Immunol 2005; 175: 5396-5402.

46. Basher F, Fan H, Zingarelli B, et al. beta-Arrestin 2: a Negative Regulator of Inflammatory Responses in Polymorphonuclear Leukocytes. Int J Clin Exp Med 2008; 1(1): 32-41.

47. Somlyo AP, Somlyo AV. Ca2+ sensitivity of smooth muscle and nonmuscle myosin II: modulated by $\mathrm{G}$ proteins, kinases, and myosin phosphatase. Physiol Rev 2003; 83: 1325-1358. 